recognised by medical staff. 27/40 (67.5\%) patients were BPS positive. Of these only $1(3.7 \%)$ was referred to HSPCT.

Conclusions This work demonstrates a significant symptom burden in patients with DCLD. This does not appear to be recognised by the medial team, meaning referral rates to HSPCT are low. Routine use of the IPOS in conjunction with BPS may aid identification and referral of patients to HSPCT, and help address this.

\section{OPIOID THERAPY IN CHRONIC CANCER PAIN - ARE WE DOING THE RIGHT THING?}

Hannah Clare, Lisa La Mola, Richard Berman. The Christie NHS Foundation Trust

\subsection{6/bmjspcare-2018-ASPabstracts. 184}

Background People are surviving cancer for longer and are undergoing more lines of treatments. Their survival may be complicated by chronic pain for which they can be on longterm opioids. The pitfalls of chronic opioid use are well documented in non-cancer pain. At The Christie, there are increasing referrals to the Supportive Care Team (SCT) for patients with uncontrolled pain, on high doses of opioids experiencing significant adverse effects, whilst being actively treated for cancer.

Aims Assess the response in pain levels, side effects and ability to remain on treatment following opioid reduction, and adoption of a more targeted approach to pain control.

Method A retrospective case note review was conducted. Those included had been referred with worsening chronic pain as the predominant symptom, and an opioid reduction within the initial management plan. Data was collected with an audit tool at the initial and subsequent visit.

Results Nine case notes were reviewed. The mean opioid reduction was 35\% (range 20\%-100\%). All patients reported a reduction in their self-rated pain score with $78 \%$ reporting at least an improvement of 2 points (out of 10). All those who felt constipated reported an improvement, and 83\% who reported drowsiness felt it had resolved. At $89 \%$ of clinic visits a non-opioid medication was either started or titrated. Breakthrough opioid doses were altered in all (one excluded as no documentation) with $50 \%$ having a dose reduction (average $37.25 \%$ ) and $62.5 \%$ starting trans-mucosal fentanyl. There were no delays in those on active treatment.

Conclusion Reducing overall opioid dose, treating breakthrough pain as a separate entity and optimising non-opioid medications improved both pain control and the adverse effects of opioids.

Early referral to the SCT can help optimise pain control, reduce side effects and allow patients to maintain their performance status to continue treatment.

\section{THE 'FORGOTTEN' GENERATION: QUALITY OF LIFE IN MEN LIVING WITH DUCHENNE MUSCULAR DYSTROPHY}

Sheonad Laidlaw, Emma Carduff. Marie Curie Care, University of Cardiff

10.1136/bmjspcare-2018-ASPabstracts. 185

Background Duchenne Muscular Dystrophy (DMD) is an $\mathrm{x}^{-}$ linked, ultra-rare neuromuscular condition affecting 1 in 3600-6000 live male births. Life expectancy is increasing thanks to targeted intervention and advancing medical treatment. Longer survival and advancing age means a greater dependence on family, friends and the wider community, and increasing social and health care costs. However, little is known about quality of life (QoL) in adults with DMD.

Aim To investigate and explore quality of life in men living with DMD in the West of Scotland.

Methods This was a qualitative interview study using the 'Schedule for the Evaluation of Individual Quality of life Direct Weighting' (SEIQoL-DW) tool, an interview based instrument which allows the assessment of elements that contribute to an individual's QoL. Men over the age of 25 and living in the West of Scotland were identified and recruited through the Scottish Muscle Network - a multidisciplinary clinical network. The interviews were transcribed and analysed thematically applying descriptive labels to the data.

Results Six men were recruited and interviewed. A number of themes were characterised as key to good QoL: strong personal relationships providing support and advocacy; meaningful connection with the world through hobbies and on-line communities; being in control of physical and mental well being, supported by knowledgeable and approachable health care professionals; continued independence through use of essential equipment both in and out of the adapted accessible home; and a positive, resilient attitude to life with a disability. Conclusions Key improvements could improve QoL in this 'forgotten' group of adults: upskilling and support for all care givers; lifelong input from physiotherapy; timely access to psychological support; improved access to respite facilities; better co-ordinated holistic multidisciplinary care; and proactive advance care planning.

\section{ENHANCED SUPPORTIVE CARE (ESC): RESULTS FROM THE NATIONAL CQUIN DATA (YEAR 1)}

Richard Berman, Lisa La Mola, Elizabeth Elliott, Nathan Hall, Jill Lockhart. NHS England, The Christie NHS Foundation Trust

\subsection{6/bmjspcare-2018-ASPabstracts.186}

Background The landscape of cancer care is changing rapidly. As more patients live longer with 'chronic cancer' and new treatments become increasingly available, there is a growing need for oncologists, palliative care specialists and acute physicians to contribute more to the development of expertise in managing problems associated with long-term cancer and cancer survival. ESC is a national cancer centre initiative which aims to implement early involvement of supportive care within oncology. The ultimate aim of ESC is to make available supportive care expertise for patients at any stage, including those with curable cancers.

Methods ESC is being delivered through a national cquin. This has encouraged additional investment allowing resource expansion required to deliver ESC. In most centres, development of ESC has been led by palliative care clinicians and incorporated within palliative care teams. In other centres, this is led by oncologists. Some 'rebranded' the name of their palliative care team to include 'supportive care' or 'symptom control'; whilst others have developed separate 'ESC teams' within their centre. In this 1st phase of ESC, referrals to supportive care are encouraged for patients who are within 6 weeks of a diagnosis of incurable cancer.

Results ESC is a 3-year national project (2016-2019). 23 cancer centres across England signed up to the initiative in 
2016-2017. Most of these sites now demonstrate earlier integration of supportive care within cancer through implementation of ESC - some have shown very significant increases in early referrals. In some sites, data points to an impact on reducing emergency admissions.

Conclusions NHS England (NHSE) continues to build the case that ESC delivers improved value - reduced healthcare costs, improved QoL and optimises treatment decision making. Data collection is ongoing nationally and will inform national cancer policy and NHSE strategy. Formal research evaluation of ESC is expected begin in early 2018.

\section{IMPLEMENTATION OF SERIOUS ILLNESS CARE PROGRAMME UK FOR PATIENTS WITH ADVANCED CANCER: FEASIBILITY STUDY}

Tamsin McGlinchey, Stephen Mason, Alison Coackley, Anita Roberts, Maria Maguire, Justin Sanders, Francine Maloney, Susan Block, John Ellershaw, Peter Kirkbride. Marie Curie Palliative Care Institute in Liverpool, Clatterbridge Cancer Centre NHS Foundation Trust, Ariadne Labs Brigham and Women's Hospital and Harvard T.H. Chan School of Public Health

\subsection{6/bmjspcare-2018-ASPabstracts. 187}

The Serious Illness Care Programme is a complex intervention developed in the US to improve communication between clinicians and patients with serious illness. In collaboration with Aridane Labs the programme has been developed for use within the UK, with pilot implementation funded by NHS England. Within the programme, clinicians are trained in use of a Serious Illness Conversation Guide (the guide), to structure conversations with patients/families. A feasibility of the UK programme is underway, within one North West cancer centre.

Aim To present interim results from the feasibility study

Methods Data on the use and experience of the UK programme, from the perspective of clinicians and patients

Quantitative Data: Questionnaires:

Clinicians - pre/post training:

- confidence;

- attitudes to care of the dying;

- acceptability of serious illness care programme

Patients - pre/post serious illness conversation:

- Quality of clinical communication;

- Quality of life;

- Anxiety;

- depression.

Qualitative Data: In depth interviews:

Clinicians: experience of the UK programme, education and training and engaging patients in serious illness conversations

Patients: experience of engaging in serious illness conversations, including their acceptability of the approach

Results Quantitative Data: Results will be presented to assess the feasibility and acceptability of the UK programme:

- Proportion of patients identified conversations and participation in study measures

- Illustrate any indicative improvements in communication and patient outcomes.

Qualitative Data: Thematic Analysis to explore perceptions and experience of the programme: results will highlight main themes, such as: Conversation Value; Holistic and Compassionate; Opening up conversations with family members; Breaking the ice; Timing of conversation and Prognosis.

Conclusion Results from this study suggest these conversations promote shared decision making and individualised care planning, and espouse 'holistic' and 'person centred' care. Further research and evaluation is required to understand the effect of the UK programme on patient outcomes.

\section{E ASSESSING THE IMPACT OF ENHANCED SUPPORTIVE CARE USING THE INTEGRATED PALLIATIVE CARE OUTCOME SCALE (IPOS) AT THE CHRISTIE NHS FOUNDATION TRUST}

Lisa La Mola, Mark Warren, Richard Berman, Shameem Lilley, Phil Higham, Hannah Clare. The Christie NHS Foundation Trust

10.1136/bmjspcare-2018-ASPabstracts. 188

Enhanced Supportive Care (ESC) is a new UK initiative that integrates supportive care within oncology. At its heart is better access to expertise in managing the adverse effects of cancer and cancer treatments to enhance the patient experience and reduce the need for hospital admission.

ESC is award winning and has been recognised nationally by NHS England.

The aim of this audit was to measure the impact of ESC using the Integrated Palliative Care Outcome Scale (IPOS). This is a validated tool that can be used in clinical audit, research and training to measure symptom burden.

Method 47 patients referred into the supportive care service, were randomly selected to complete the IPOS tool within 3 areas (inpatients, outpatients and trials unit) during May to October 2016. For all areas a baseline IPOS was completed prior to the initial assessment by supportive care. Subsequent IPOS were completed approximately 3 days later for inpatients. Outpatients and trial patients were reviewed at various time points depending on the timing of their follow up appointments. Outpatients had an average follow up of 41 days. Trial patients had an average follow up of 15 days.

Results The overall IPOS score is the sum of the score of each of the 17 questions and can range from 0-68. A reduction in the IPOS score demonstrates an improvement in physical and psychological patient symptom burden. 16 inpatients completed the IPOS and demonstrated a 50\% improvement in overall mean symptom score. 5 experimental cancer medicine patients completed the IPOS and demonstrated a $49 \%$ improvement in overall mean score. 26 oncology outpatients completed the IPOS and demonstrated a $2.5 \%$ deterioration in overall mean score, demonstrating a possible correlation between frequency of supportive care review and symptom burden.

\section{UK AND IRISH HOSPICE AND SPECIALIST PALLIATIVE CARE UNIT EXPERIENCE OF ASSISTED VENTILATION IN MOTOR NEURONE DISEASE}

Aoife Gleeson, Faye Johnson. Aneurin Bevan University Health Board

10.1136/bmjspcare-2018-ASPabstracts.189 